\title{
Evaluating physician awareness of common health care costs in the emergency department
}

\author{
Rohit Gandhi, $\mathrm{MD}^{*}{ }^{\dagger}$; Ian Stiell, MD, MSc ${ }^{\ddagger}$; Alan Forster, MD, MSc*; James Worthington, MD*; \\ Madeleine Ziss, $\mathrm{MD}^{*}$; Jack B. Kitts, MD, MBA*; Ranjeeta Malik, PhD*
}

\section{ABSTRACT}

Background: Health care costs are on the rise in Canada and the sustainability of our health care system is at risk. As gatekeepers to patient care, emergency department (ED) physicians have a direct impact on health care costs. We aimed to identify current levels of cost awareness among ED physicians. By understanding the current level of physician cost awareness, we hope to identify areas where cost education would provide the greatest benefit in reducing ordering costs.

Methods: We conducted a survey evaluating current awareness of common ordering costs among ED physicians from two tertiary teaching hospitals. Our study population was comprised of 124, certified emergency medicine staff physicians and emergency medicine resident physicians. Our survey asked ED physicians to estimate the costs of 41 items across four categories of day-to-day ordering: imaging investigations, materials, laboratory tests, and pharmaceuticals. Items were selected based on frequency of use, availability of cost-effective alternatives, and tests considered to be "low yield". The primary outcome was percentages of underestimates, correct estimates, and overestimates for ED costs among ED physicians.

Results: The average percentage of correct cost estimates among ED physicians was $14 \%$ across the four ordering categories. Where cost-effective alternatives exist, ED physicians overestimated the cost of the more cost-effective item. They also underestimated the cost of low-yield tests.

Interpretation: ED physicians demonstrated limited cost awareness of common health care costs. Further studies that characterize utilization of hospital resources based on ED physician awareness of cost-effective alternatives and cost of "low yield" tests are needed.

\section{RÉSUMÉ}

Contexte: Le coût des soins de santé augmente au Canada, ce qui compromet la pérennité du système de soins de santé. Les urgentologues, en tant que professionnels de première ligne, ont une incidence directe sur le coût des soins de santé. Aussi avons-nous tenté d'évaluer le degré de connaissance du coût de diverses demandes par les urgentologues. Ce faisant, nous espérons cerner les domaines où une formation en la matière se traduirait par les plus fortes réductions de coût.

Méthode: Une enquête visant à évaluer le degré de connaissance du coût de demandes courantes par les urgentologues a été menée dans deux hôpitaux universitaires de soins tertiaires. La population à l'étude se composait de 124 urgentologues, membres du personnel, titulaires d'un certificat de compétences, et de résidents en médecine d'urgence. Les urgentologues devaient estimer, dans le questionnaire, le coût de 41 unités provenant de quatre catégories de demandes courantes, soit les examens par imagerie, le matériel, les examens de laboratoire et les médicaments. Le choix des unités reposait sur la fréquence d'utilisation, I'existence de solutions de rechange rentables et le faible degré de rendement de certains examens. Le principal critère d'évaluation consistait en les pourcentages de sous-évaluation, d'évaluation juste et de surévaluation du coût de certaines demandes faites par les urgentologues.

Résultats: Le pourcentage moyen des évaluations justes du coût des unités a atteint $14 \%$, et ce, dans l'ensemble des quatre catégories. Dans les cas où il existait des solutions de rechange rentables, les urgentologues ont surestimé le coût des unités plus rentables. Ils ont toutefois sous-évalué le coût des examens jugés à faible rendement.

Interprétation: Les urgentologues ont fait preuve d'une méconnaissance du coût des soins de santé courants. II faudrait mener d'autres études qui caractérisent l'utilisation des ressources hospitalières, fondée sur la connaissance qu'ont les urgentologues des solutions de rechange rentables et du coût des examens à faible rendement.

Keywords: emergency department, cost savings, cost awareness, healthcare costs

\section{INTRODUCTION}

Healthcare costs are on the rise in Canada, and the sustainability of our healthcare system is at risk. Healthcare in Canada is delivered through a group of

From the *Ottawa Hospital, Ottawa, ON; †Department of Medicine, University of Ottawa, Ottawa, ON; and the $¥$ Department of Emergency Medicine, Clinical Epidemiology Program, Ottawa Hospital Research Institute, Ottawa, ON.

Correspondence to: Dr. Rohit Gandhi, 55 Hyannis Avenue, Ottawa K2J 2W9, Ontario; Email: rohit.gandhi@uottawa.ca 
publically funded health insurance plans that provide all Canadian citizens with universal coverage. Funding is administered by individual provinces and territories based on guidelines set by the federal government. Over the last 15 years, total federal healthcare expenditures have more than doubled from just under $\$ 100$ billion in 2001 to $\$ 215$ billion in 2014. ${ }^{1}$ The Canadian Society of Actuaries suggested that at current growth rates $97 \%$ of total revenues available to Canadian provinces and territories would be spent on healthcare by 2037 , compared to $44 \%$ in $2012 .^{2}$ As the Canadian population ages, a growing number of patients are seeking immediate and unscheduled medical care through our emergency departments (EDs). The ED is an expensive alternative to other forms of primary care, and Canadians are among the most frequent users of EDs worldwide. ${ }^{3-5}$ Given these trends, physicians and hospital administrators must identify innovative and cost-efficient approaches to healthcare delivery.

Previous studies have discussed system-level changes to help control costs, but physician-level solutions have not been well-explored. As gatekeepers to patient care, ED physicians have a significant and direct influence on the cost of healthcare. ${ }^{6}$ However, the Canadian healthcare system is not designed to encourage physicians to manage resources and costs. A curriculum review of Canadian medical schools reveals no formal training on healthcare costs. Several studies in the United States, Israel, and Sweden have shown that physicians respond to increased cost awareness by significantly reducing the number of investigations ordered, which results in significant cost savings. ${ }^{7-12}$ In over a decade, there have been no Canadian studies evaluating ED physician cost awareness across the four main categories of day-to-day ordering: imaging investigations, materials, laboratory tests, and pharmaceuticals. Older Canadian studies demonstrate poor cost awareness among ED physicians. ${ }^{13-15}$ More recent studies from the United States show a similar trend. ${ }^{16}$ The recent advent of electronic medical systems can allow for access to cost information at the point of care, which could be used to facilitate cost-conscious clinical decision making. ${ }^{17,18}$ A recent study of residents at an academic hospital in the United States demonstrated that by incorporating cost information into electronic medical records, residents were more likely to estimate the costs of laboratory tests correctly. ${ }^{19}$ With a growing number of high-cost diagnostic tools and pharmaceutical options at the disposal of ED physicians, ${ }^{20,21}$ differences in cost could become an important consideration when choosing between similar options. Given the demonstrated deficits in physician awareness of healthcare costs in prior studies, we aim to characterize current levels of cost awareness among ED physicians at our center. By understanding the current level of physician cost awareness, we hope to identify areas where cost education would provide the greatest benefit in reducing ordering costs at our hospital and indirectly reduce costs to the healthcare system.

\section{METHODS}

\section{Study design and setting}

We conducted a cross-sectional survey evaluating current awareness of common ordering costs among ED physicians-both department staff and emergency medicine residents-from two campuses of a tertiarycare teaching hospital in Ottawa, Ontario. The two EDs have an annual patient census of 150,000 visits and serve as regional centres for cardiac care, trauma, stroke, and cancer.

\section{Study population}

Our survey population was made up of 124 certified emergency medicine staff physicians and emergency medicine resident physicians. Approval from The Ottawa Hospital (TOH) Research Institute Ethics Board was obtained (protocol no. 20140871).

\section{Data collection}

We considered ED costs as subdivided into four categories: imaging investigations, materials, laboratory tests, and pharmaceuticals. Cost information for items in these categories was collected from the finance and performance measurement departments at TOH. Costs are reported in 2014 Canadian dollars. For the purposes of the present study, actual costs were defined as "total direct costs" to TOH. "Total direct" costs included "fixed" costs (e.g., overhead, technician salary) and "direct variable" costs (e.g., reagents). They did not include any "indirect" costs (e.g., administration) or physician billings (e.g., radiologist interpretation). Laboratory tests and pharmaceuticals did not include collection materials (e.g., test tubes) or medication delivery materials (e.g., needles). 
A total of 41 items across each of the four categories (imaging investigations, materials, laboratory tests, and pharmaceuticals) were selected for the survey based on high frequency of use, availability of cost-effective alternatives, and tests considered to be "low-yield." The survey was created using Survey Monkey's online platform and distributed via email to ED staff physicians and resident physicians by the chief of the department. Three reminder emails were sent over a two-month period. Participants were asked to estimate the cost of the 41 items and input their estimates using "free-text" fields that allow entry of values precise to the cent. An explanation of "total direct costs" was provided for each category. Participants were asked not to include indirect costs or physician billings. The survey could be exited at any time, but questions could not be skipped, and the data were only collected once participants submitted a fully completed survey.

\section{Outcome measures}

The primary outcome was percentage of correct estimates, underestimates, and overestimates of cost across the four categories of day-to-day ordering among ED physicians. Correct estimates are defined as estimates within $25 \%$ above or $25 \%$ below actual cost, overestimates are defined as estimates greater than $25 \%$ above the upper limit of actual cost, and underestimates are defined as estimates more than $25 \%$ below the lower limit of the actual cost. Specifically, we were interested in the percentages of correct estimates, underestimates, and overestimates of the cost of items with cost-effective alternatives and low-yield tests.

\section{Analysis}

The percentage of underestimates, correct estimates, and overestimates was reported for each item. Data were analyzed using descriptive analyses as appropriate for the type of data. Data analysis was performed using SAS and Microsoft Excel ${ }^{\circledR}$.

\section{RESULTS}

The survey was distributed to 124 ED staff physicians and resident physicians by email. Our response rate was $58 \%$. Of the $72 \mathrm{ED}$ physicians who responded to the survey, 43 were ED staff physicians and 29 were ED resident physicians. The participants provided 2,952

\begin{tabular}{lc} 
Table 1. Survey participant demographics & \\
\hline & $n(\%)$ \\
Gender & $(\mathrm{n}=72)$ \\
\hline Male & $47(65)$ \\
Female & $25(35)$ \\
Position & \\
Staff physician & $43(60)$ \\
Resident physician & $29(40)$ \\
Designation or designation in training & \\
CCFP-EM & $23(32)$ \\
FRCPC & $47(65)$ \\
ABEM (American Board) & $2(3)$ \\
Years in practice (staff only) & \\
$<2$ & $8(21)$ \\
$2-5$ & $6(14)$ \\
$6-15$ & $12(28)$ \\
16-25 & $9(21)$ \\
>25 & $7(16)$ \\
Would better access to cost information affect parts of your ED \\
practice? \\
Yes \\
Likely \\
Unsure \\
Unlikely \\
No & $22(31)$ \\
\end{tabular}

cost estimates for 41 items. Participating physicians answered each survey question, and no data were missing (a completion rate of $100 \%$ ).

There was no significant difference in correct estimates between ED staff and ED residents $(\mathrm{p}=0.64)$ or between staff with $\leq 5$ years of experience and staff with $>5$ years of experience $(\mathrm{p}=0.23)$.

Table 1 describes the survey participant demographics and the percentage of participants who answered "yes," "likely," "unsure," "unlikely," or "no" to the question "Would better access to cost information impact your practice?" The majority of participants (67\%) answered "likely" or "yes," and no participants (0\%) answered "no."

Figure 1 provides an overview of the percentage of correct estimates, overestimates, and underestimates for each of the four categories. The percentage of correct cost estimates among ED physicians did not exceed 25\% in any of the four categories. The pharmaceuticals category was the most overestimated at $88 \%$ and was also the least accurate, with $4 \%$ accurately estimated costs.

Table 2 provides actual cost information as well as the median, range, and interquartile range (IQR) for ED physician cost estimates and the percentage of 
Table 2. Proportion of physician underestimates, correct estimates, and overestimates

\begin{tabular}{|c|c|c|c|c|c|}
\hline \multirow[b]{2}{*}{ Imaging investigations } & \multirow[b]{2}{*}{ Actual cost, $\$$} & \multicolumn{2}{|c|}{$\begin{array}{c}\text { Physician estimates, } \$ \\
\text { ( } \mathrm{n}=72)\end{array}$} & \multirow[b]{2}{*}{ IQR } & \multirow[b]{2}{*}{$\%$ correct estimates ${ }^{*}$} \\
\hline & & Median & Range & & \\
\hline CT head: no contrast & 45.79 & 200 & $40-1500$ & 100-300 & 7 \\
\hline CT: pulmonary embolism & 93.18 & 325 & $50-2000$ & $200-500$ & 10 \\
\hline MRI: limited spine 3S & 210.63 & 525 & $100-3500$ & $300-1000$ & 15 \\
\hline U/S: leg vein $\times 1$ & 89.72 & 100 & $10-500$ & 80-200 & 46 \\
\hline U/S: abdomen + pelvis & 137.36 & 200 & $10-750$ & $100-300$ & 18 \\
\hline X-ray: abdomen/KUB (2 view) & 60.71 & 50 & $10-300$ & $30-80$ & 31 \\
\hline X-ray: chest (2 view) & 26.42 & 40 & $10-300$ & $25-75$ & 33 \\
\hline X-ray: Lumbosacral spine (2-3 view) & 56.61 & 50 & $10-300$ & $30-100$ & 26 \\
\hline \multicolumn{6}{|l|}{ Materials } \\
\hline Sutures 4.0 polybutester (monofilament) & 4.09 & 4 & $1-65$ & $2.75-10$ & 26 \\
\hline Sutures 4.0 nylon (monofilament) & 1.42 & 5 & $1-65$ & 3-10 & 0 \\
\hline Lumbar puncture needle, regular & 1.42 & 10 & $0.75-75$ & $5-20$ & 0 \\
\hline Lumbar puncture needle, atraumatic & 10.25 & 11 & $0.75-100$ & $7-25$ & 29 \\
\hline Topical skin adhesive & 21.39 & 15 & $1-65$ & $6.5-20$ & 31 \\
\hline Skin Stapler & 3.88 & 25 & $2-200$ & $15-42.5$ & 0 \\
\hline Lidocaine $1 \%$ + epinephrine (20 ml) & 6.84 & 10 & $1-50$ & $5-20$ & 6 \\
\hline Adjustable cervical spine collar & 68.00 & 60 & $10-200$ & $40-90$ & 28 \\
\hline Plaster roll $10 \mathrm{~cm} \times 4.5 \mathrm{~m}$ & 2.88 & 5 & $0.5-40$ & $3-10$ & 11 \\
\hline \multicolumn{6}{|l|}{ Laboratory tests } \\
\hline $\mathrm{CBC}$ with differential & 7.35 & 10 & $0.5-100$ & $5-16.5$ & 3 \\
\hline CHEM-7 & 11.41 & 10 & $1-100$ & $5-17.5$ & 21 \\
\hline INR & 7.35 & 6.5 & $1-50$ & $4-10$ & 8 \\
\hline PTT & 7.35 & 5 & $0.25-50$ & $2-10$ & 6 \\
\hline Chloride & 1.63 & 3 & $0.05-20$ & $2-5$ & 21 \\
\hline Urinalysis: R\&M & 1.63 & 5 & $0.05-100$ & $2.5-13.5$ & 11 \\
\hline Urine culture & 18.40 & 15 & $2-150$ & $10-30$ & 33 \\
\hline TSH & 1.63 & 10 & $1-50$ & $5-15$ & 7 \\
\hline Blood culture & 18.40 & 25 & $1-200$ & $15-37.5$ & 19 \\
\hline D-dimer & 7.35 & 10 & $1-200$ & $5.5-22.5$ & 11 \\
\hline Troponin (Tnl) & 5.51 & 10 & $0.5-100$ & $5-20$ & 15 \\
\hline Creatinine kinase & 1.63 & 5 & $0.25-50$ & $2-10$ & 14 \\
\hline \multicolumn{6}{|l|}{ Pharmaceuticals } \\
\hline Ondansetron 4 mg IV & 0.60 & 10 & $0.5-100$ & $5-20$ & 3 \\
\hline Dimenhydrinate $50 \mathrm{mg}$ IV & 0.79 & 3 & $0.5-50$ & $1-5$ & 1 \\
\hline Naproxen 500 mg PO & 0.06 & 1 & $0.09-20$ & $1-2.5$ & 0 \\
\hline Ketorolac $30 \mathrm{mg} \mathrm{IM}$ & 3.50 & 5 & $0.5-75$ & $3-10$ & 13 \\
\hline Morphine $10 \mathrm{mg}$ PO & 0.17 & 2 & $0.01-30$ & $1-3$ & 1 \\
\hline Morphine $5 \mathrm{mg} \mathrm{IV}$ & 0.70 & 5 & $0.5-30$ & $2-10$ & 0 \\
\hline Hydromorphone 2 mg PO & 0.22 & 2 & $0.1-100$ & $1-5$ & 6 \\
\hline Hydromorphone 1 mg IV & 0.89 & 5 & $0.5-100$ & $2-10$ & 11 \\
\hline Levofloxacin PO $750 \mathrm{mg}$ & 0.34 & 5 & $0.4-100$ & $4-10$ & 1 \\
\hline Levofloxacin IV $750 \mathrm{mg}$ & 13.59 & 20 & $1-500$ & $10-32.5$ & 6 \\
\hline Salbutamol $100 \mu \mathrm{g} \operatorname{lnh}$ & 2.75 & 5 & $0.05-75$ & $1.5-12$ & 3 \\
\hline Ipratropium $20 \mu \mathrm{g}$ Inh & 18.92 & 5 & $0.05-75$ & $2-15$ & 13 \\
\hline
\end{tabular}


correct estimates for each item surveyed. There were some notably high overestimates among these itemsfor example, a CT head without contrast was estimated to cost $\$ 1,500$ (3,276\% higher than the actual price). Notably low estimates were also seen-for example, an ipratropium inhaler was estimated to cost $\$ 0.05$ $(37,840 \%$ lower than the actual price).

Table 3 highlights cost-effective alternatives. It outlines the absolute and relative cost differences between comparable items.

Figure 2 shows a breakdown of correct estimates, overestimates, and underestimates for each item in the imaging category. The cost of most imaging items was overestimated by ED physicians, but the cost of "X-ray: abdomen/KUB" and "X-ray: lumbosacral spine" were notably underestimated.

Figure 3 shows a breakdown of correct estimates, overestimates, and underestimates for each item in the materials category. Of note, $56 \%$ underestimated the cost of topical skin adhesive, while over $47 \%$ underestimated the cost of cervical spine collars.

Figure 4 shows a breakdown of correct estimates, overestimates, and underestimates for each item in the laboratory tests category. The costs of the CBC and differential, CHEM-7, INR, PTT, and urine culture were underestimated by a large percentage of physicians compared to other tests in this category.

Figure 5 shows a breakdown of correct estimates, overestimates, and underestimates for each item in the pharmaceuticals category. As mentioned, ED physicians grossly overestimated the cost of most items. Exceptions included IV levofloxacin, salbutamol, and ipratropium, where a large percentage of physicians underestimated the cost. Some $99 \%$ of ED physicians overestimated the cost of PO levofloxacin, and none underestimated its cost. Similarly, 22\% of cost estimates for IM ketorolac were underestimates, while $100 \%$ of estimates for the cost of PO naproxen were overestimates.

Figures 6-9 depict the range of physician cost estimates compared to the actual cost for each item across all four ordering categories. These figures highlight the large range of physician to physician cost estimates.

\section{DISCUSSION}

\section{Interpretation of results}

We discovered that ED physicians at our two tertiary-care teaching hospitals in Ottawa had limited cost awareness

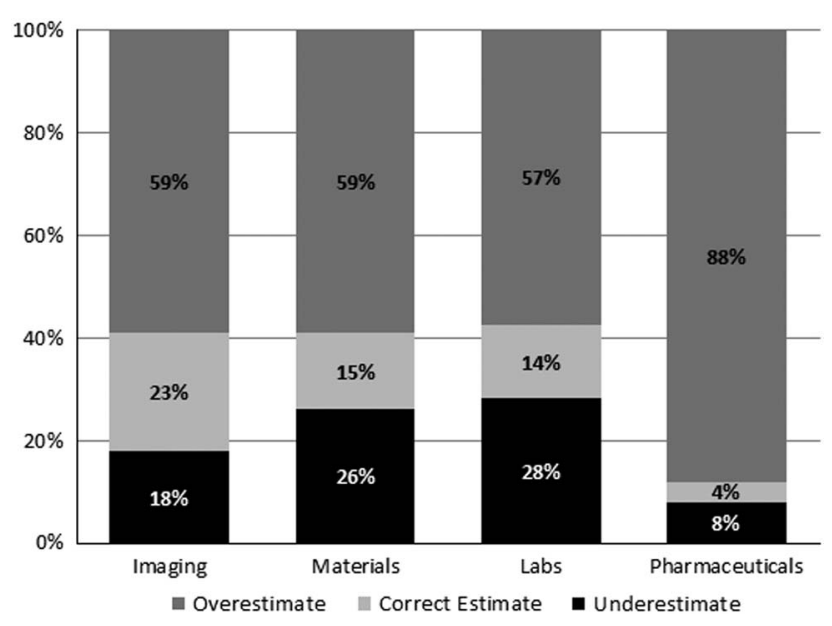

Figure 1. Percentages of ED physician under-, correct, and overestimates for cost across the four categories surveyed.

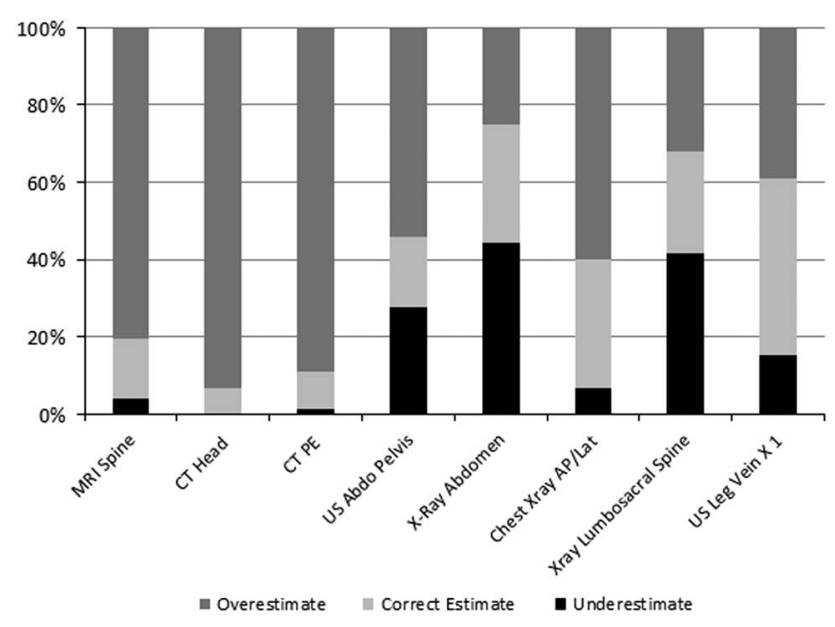

Figure 2. Percentages of ED physician under-, correct, and overestimates for cost of various imaging investigations.

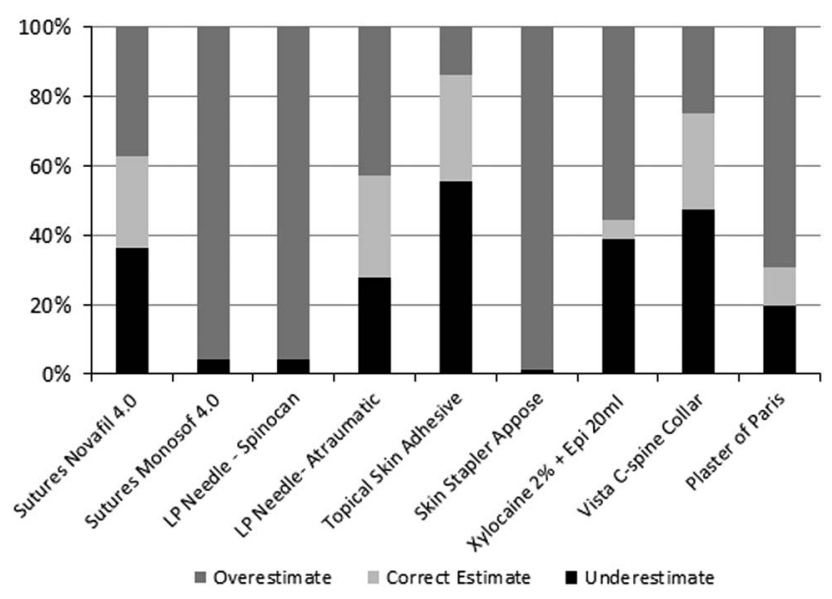

Figure 3. Percentages of ED physician under-, correct, and overestimates for cost of various materials. 


\begin{tabular}{|c|c|c|c|}
\hline \multicolumn{2}{|c|}{ Comparable items } & \multirow[b]{2}{*}{ Relative cost reduction, $\%$} & \multirow[b]{2}{*}{ Absolute cost reduction, $\$$} \\
\hline Higher-cost item & Lower-cost item & & \\
\hline Sutures 4.0 polybutester & Sutures 4.0 nylon & 65 & 2.67 \\
\hline LP Needle, atraumatic & LP Needle, regular & 99 & 8.83 \\
\hline Urine culture & Routine urinalysis & 91 & 16.77 \\
\hline Dimenhydrinate $50 \mathrm{mg}$ IV & Ondansetron $4 \mathrm{mg}$ IV & 24 & 0.19 \\
\hline Levofloxacin $750 \mathrm{mg}$ IV & Levofloxacin $750 \mathrm{mg}$ PO & 97 & 13.25 \\
\hline Hydromorphone 1 mg IV & Hydromorphone $2 \mathrm{mg} \mathrm{PO}$ & 75 & 0.67 \\
\hline Morphine $5 \mathrm{mg} \mathrm{IV}$ & Morphine $10 \mathrm{mg} P O$ & 76 & 0.53 \\
\hline Ketorolac $30 \mathrm{mg} \mathrm{IM}$ & Naproxen 500 mg PO & 98 & 3.44 \\
\hline
\end{tabular}

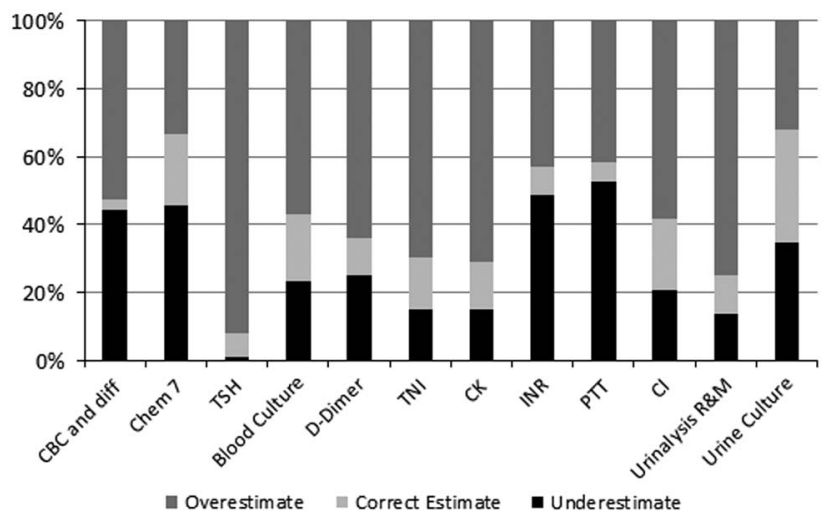

Figure 4. Percentages of ED physician under-, correct, and overestimates for cost of various laboratory tests.

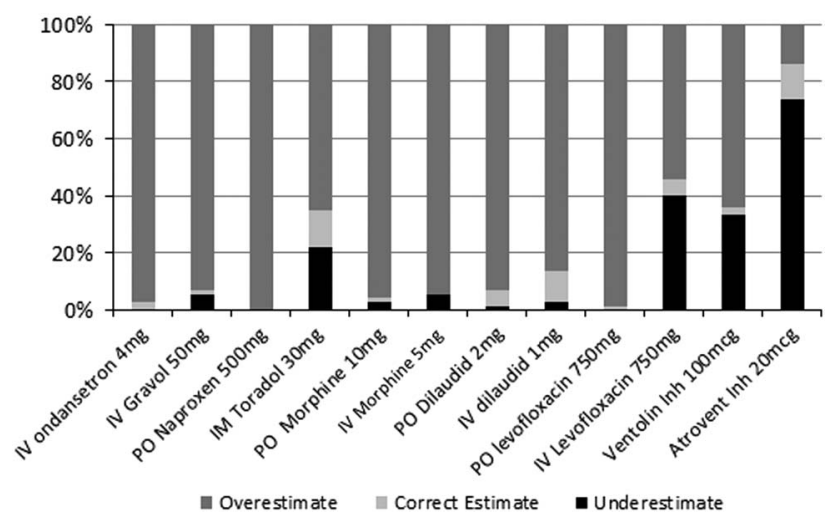

Figure 5. Percentages of ED physician under-, correct, and overestimates for cost of various pharmaceuticals.

with regard to imaging investigations, materials, laboratory tests, and pharmaceuticals. Among the four categories surveyed, imaging investigation costs had the greatest number of correct cost estimates, but also the greatest range of cost estimates. Pharmaceuticals had the smallest percentage of correct cost estimates.
Most ED physicians in our study overestimated costs across all four categories. As with most industries, cost overestimation has been shown to decrease utilisation of resources in healthcare. ${ }^{22}$ Thus, greater cost awareness in the setting of physicians who tend to overestimate cost could create an unintended increase in resource utilisation across all four categories. However, we believe that the overestimation seen in our results could be attributed to selective perception and impact biases. By conducting a survey of hospital costs, the participants' perceptions of our hypothesis might be that physicians undervalue the healthcare costs. This creates a "selective perception bias" whereby the expectation that physicians undervalue healthcare costs changes the participant's perception, leading to an overcorrection of cost estimates. An "impact bias" could also contribute to these survey findings; participants are biased toward overestimating cost because that is the more acceptable alternative to undervaluing cost, which is regarded as being less resource-conscious.

\section{Previous studies}

Overall, our findings are in keeping with previous studies, ${ }^{13,14,23}$ suggesting that physicians continue to lack cost awareness of the care they provide. Only 14\% of all cost estimates across the four categories were considered correct, which is lower than expected compared to similar studies. Innes et al. ${ }^{23}$ also considered estimates within 20 or $25 \%$ of the actual cost to be "good knowledge" and reported 25-47\% of ED physician cost estimates to be correct. The low percentage of correct cost estimates found in our study may be reflective of the rising number of available and advanced imaging investigations, materials, laboratory tests, and pharmaceuticals available to ED physicians. 


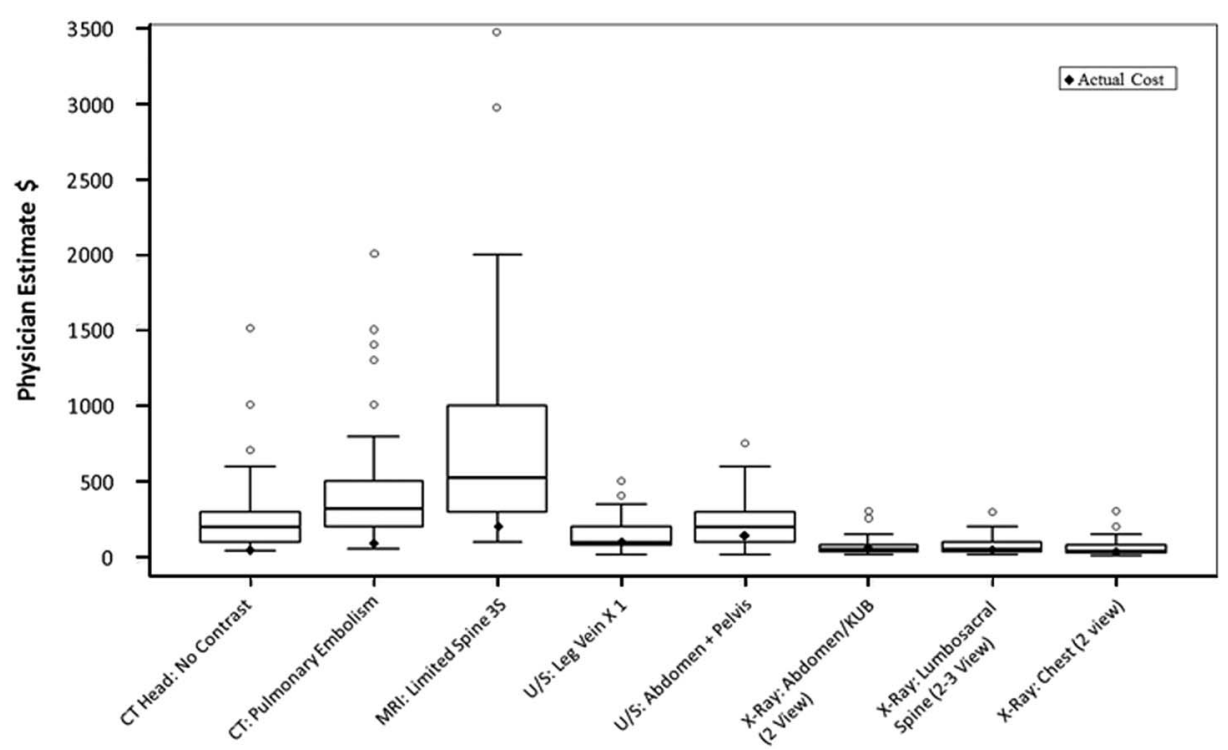

Figure 6. ED physician cost estimates of various imaging investigations.

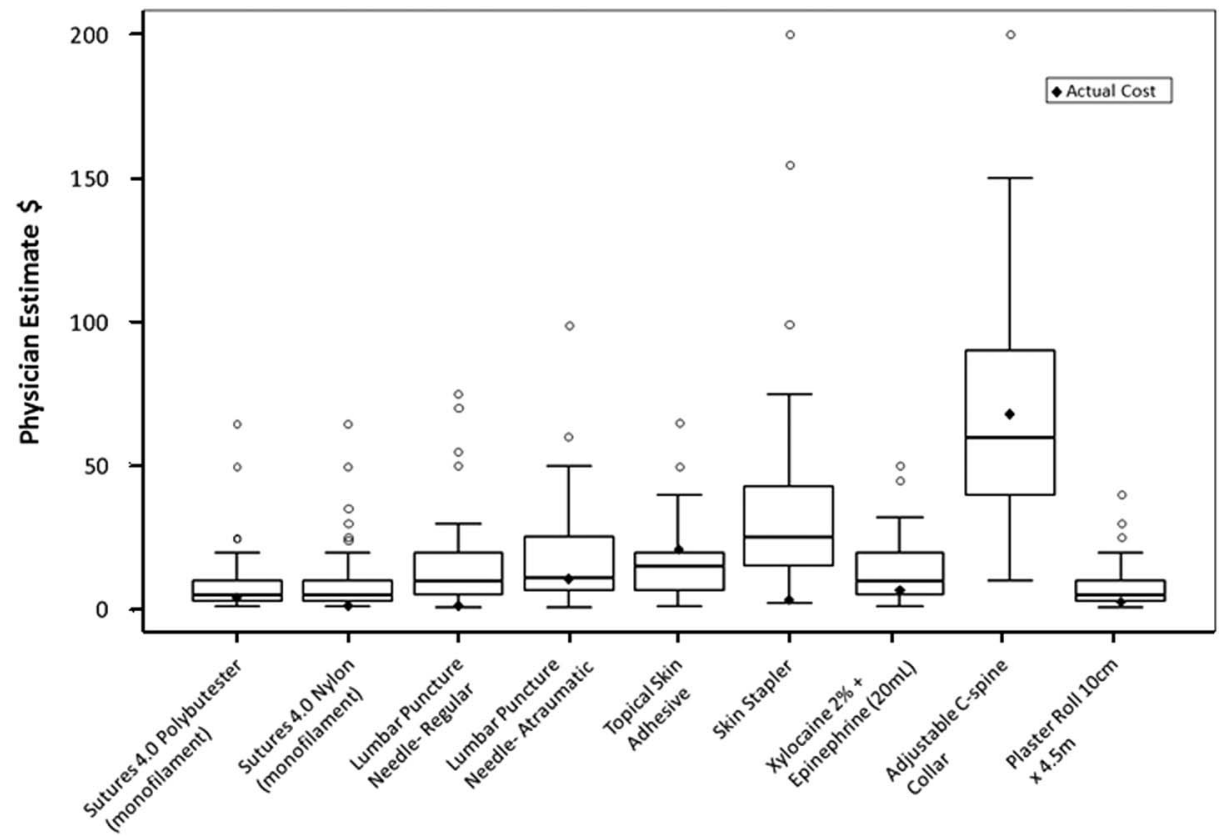

Figure 7. ED physician cost estimates of various materials.

\section{STRENGTHS}

Our study provides an updated analysis of ED physician awareness of the common ordering costs of all major ED cost categories across two tertiary-care teaching hospitals. It is the first study to investigate cost awareness across the four main categories of day-to-day ordering and the first to evaluate ED physician cost awareness of cost-effective alternatives.

\section{LIMITATIONS}

The generalizability of our study results is limited given the modest sample size of academic emergency staff and residents and the $58 \%$ response rate. While the costs were specific to our urban hospital network, the large variance in physician cost estimates at our centre would support the need for increased cost awareness irrespective of actual costs at other centers. 


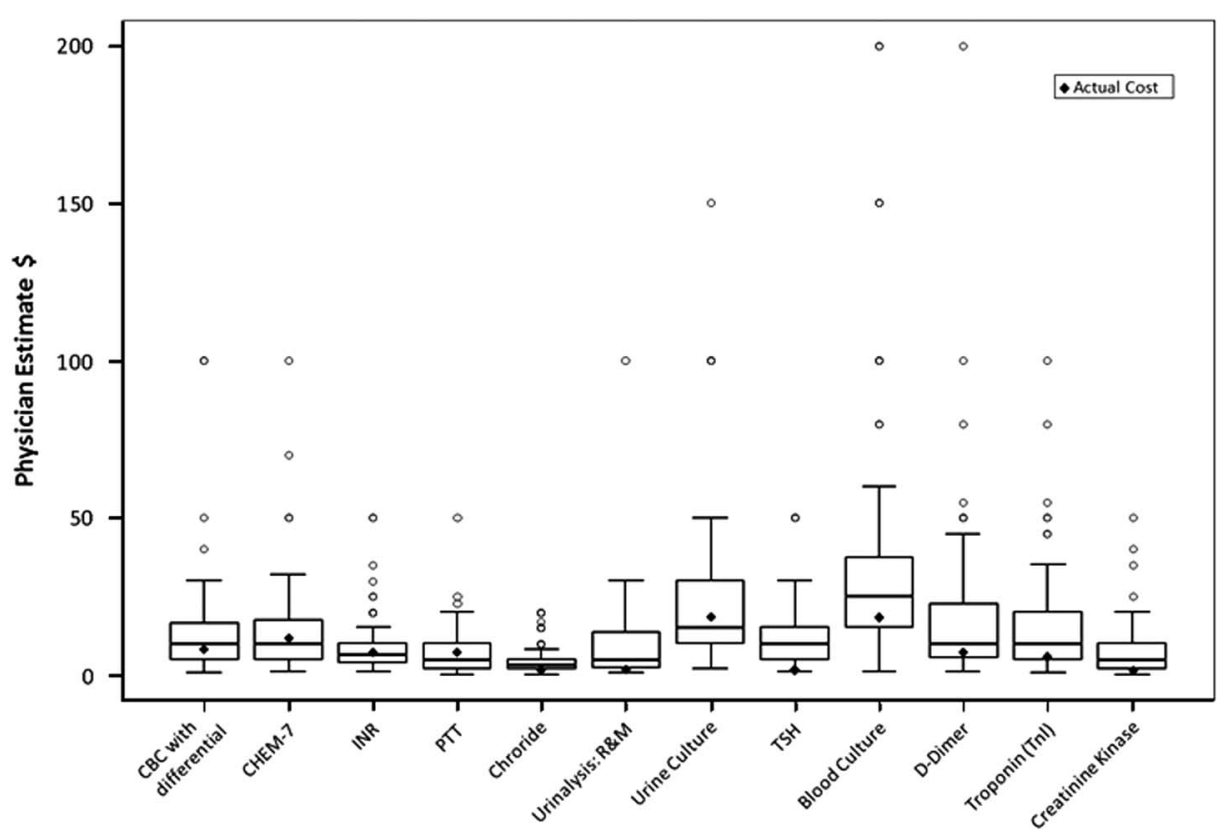

Figure 8. ED physician cost estimates of various laboratory tests.

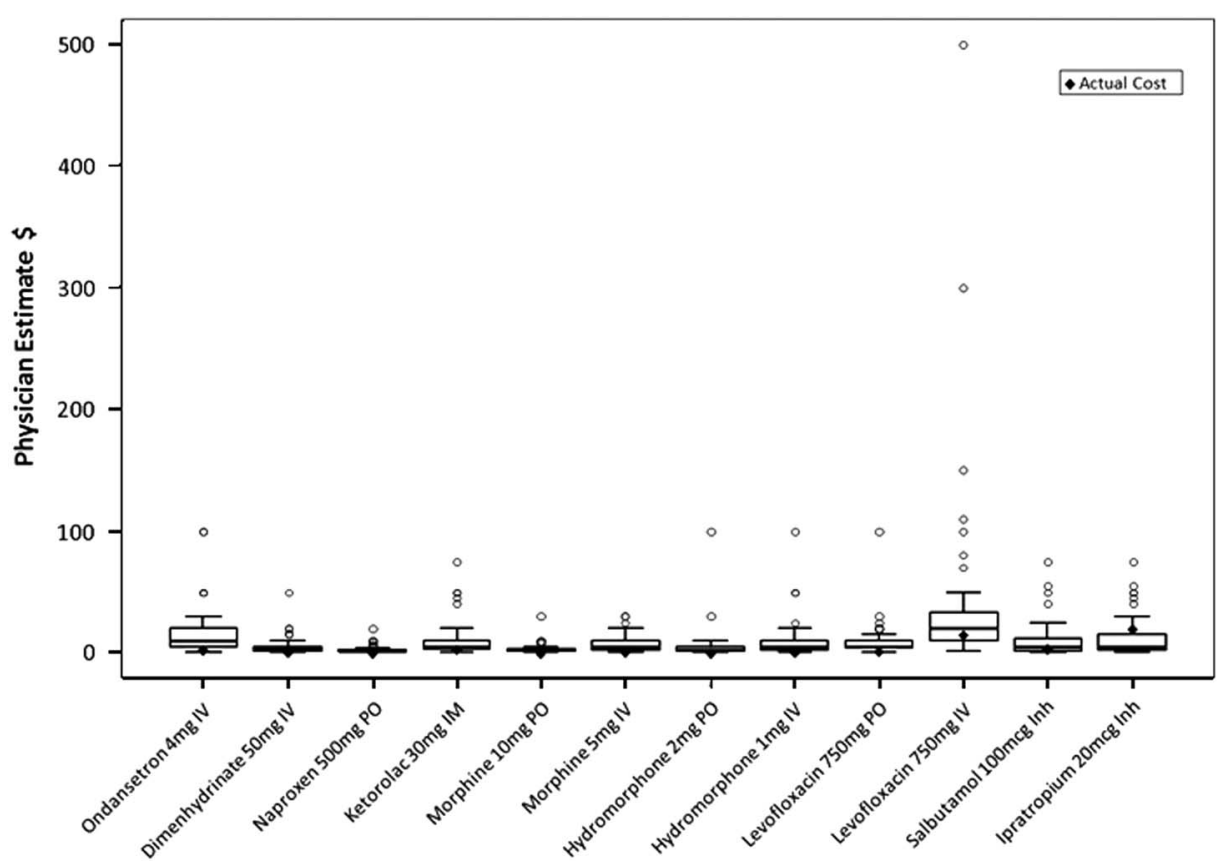

Figure 9. ED physician estimates of various pharmaceuticals.

In addition, total direct costs were used to represent "actual costs" in our study. This excludes indirect costs associated with each item, which significantly underestimates the true total cost. However, by removing indirect costs from our study, respondents were able to estimate a more tangible component of cost, and our finance department was able to provide a more accurate figure for the cost of each item. While there is no guarantee that participants did not research pricing information while responding to the survey, this information is difficult to obtain. Prices are unique to our hospital, with no such data available publically or internally without formal written requests. Finally, as mentioned in our interpretation of the results, the data 
were subject to selection perception and impact biases, which potentially resulted in overestimation of costs.

\section{CLINICAL IMPLICATIONS}

Our study highlights two areas where cost education programs might influence ordering behaviour: 1) items with cost-effective alternatives and 2) low-yield tests. Both are areas in which improved cost education with regard to only a few items may influence ordering habits on a large scale, which could result in significant cost savings without impacting patient care.

Where comparable items exist (see Table 3), ED physicians tended to overestimate the cost of the more cost-effective alternative. For example, levofloxacin, a commonly used antibiotic, has near identical bioavailability through the PO route compared to $\mathrm{IV} .^{24}$ The perceived cost of the IV formulation of levofloxacin was largely underestimated compared to the equivalent PO dose, which was significantly overestimated. The absolute cost reduction for the equivalent dosing was $\$ 13.25$ per dose. Similarly, the more cost-effective PO route for morphine and hydromorphone were overestimated to a greater degree than their equivalent IV dose. The absolute cost reduction for a single $\mathrm{PO}$ versus IV dose was $\$ 0.53$ for morphine and $\$ 0.67$ for hydromorphone. When comparing naproxen and ketorolac, two common nonsteroidal anti-inflammatory drugs (NSAIDs), our study demonstrated a relative overestimation of the inexpensive naproxen compared to ketorolac. The absolute cost reduction for equivalent dosing was $\$ 3.44$ per dose. Studies have demonstrated no significant difference between the two NSAIDs. ${ }^{25}$ These findings highlight an area where cost education can assist physicians in choosing the cost-effective alternative from among the clinically equivalent options.

Several items in the survey were selected for their increased ordering frequency, as well as for their lowyield diagnostic utility. Interestingly, ED physicians underestimated the cost of some of these items. For example, lumbosacral $\mathrm{X}$-rays are frequently ordered in the ED, yet their diagnostic utility in the majority of patient presentations is poor. ${ }^{26}$ Coagulation screens ordered in adults often include partial thromboplastin time (PTT), which, despite limited diagnostic value outside of heparinized patients or those with known bleeding disorders, are routinely performed with prothrombin times (PTs). Urine microscopies are often used to screen for urine infections. A negative urine microscopy is unlikely to yield a positive urine culture; however, urine cultures are commonly ordered in conjunction with the initial urine microscopy. If physicians delayed ordering urine cultures unless a urine microscopy was positive, an absolute cost reduction of $\$ 16.77$ per test would be realized for each negative urine microscopy. Increasing physician awareness of the costs of these three low-yield investigations could prove to be an important area of waste reduction. Taken together, these findings suggest that those accountable for ED spending and utilization should perform their own practice and cost review and educate their physicians on areas where the greatest impact to cost savings can be achieved.

\section{RESEARCH IMPLICATIONS}

Developing and evaluating cost-awareness education programs may be an effective way to mitigate the rise of healthcare costs in Canada. Further studies that characterize utilization of hospital resources based on ED physician awareness of cost-effective alternatives and the cost of "low-yield" tests would help better characterize the need for educational intervention. Studies that evaluate cost utility and resource utilization at other centres across Canada are also warranted.

\section{CONCLUSIONS}

Canadian ED physicians demonstrated limited awareness of common healthcare costs used in day-to-day practice. Our study identifies two areas where cost education programs might influence ordering behaviour: 1) items with cost-effective alternatives and 2) low-yield tests. ED physicians tended to greatly overestimate the cost of the less-expensive option among items with comparable alternatives and underestimated the cost of low-yield testing modalities. Overall, this study highlights the need for costawareness education programs and better access to cost information that would equip physicians to manage cost and hospital resources more effectively.

Acknowledgements: RG participated in the design of the study, conducted the background literature review, managed the research ethics board application, collected the data, participated in the analysis and interpretation of the data, and wrote the final manuscript. Supervising Drs. JW, AF, and IS assisted with the 
design of the study and provided guidance throughout data collection and interpretation. MZ contributed to analysis and interpretation of data and critically revised the final manuscript. Data analysis was conducted by RM at The Ottawa Hospital Research Institute.

\section{REFERENCES}

1. Canadian Institute for Health Information. National Health Expenditure Trends, 1975 to 2014. Ottawa: Canadian Institute for Health Information; 2014. Available at: https:// www.cihi.ca/en/nhex_2014_report_en.pdf (accessed June 4, 2017).

2. Canadian Institute of Actuaries and Society of Actuaries. Sustainability of the Canadian Health Care System and Impact of the 2014 Revision to the Canada Health Transfer; 2013. Available at: https://www.soa.org/research-reports/ 2013/Sustainability-of-the-Canadian-Health-Care-Systemand-Impact-of-the-2014-Revision-to-the-Canada-HealthTransfer/ (accessed June 4, 2017).

3. Statistics Canada. Projected Population, by Projection Scenario, Sex and Age Group as of July 1, Canada, Provinces and Territories, Annual. CANSIM Table 052-0005. Ottawa: Statistics Canada; 2011. Available at: http://www5.statcan. gc.ca/cansim/a26?lang=eng\&id=520005 (accessed June 4, 2017).

4. Auditor General of Ontario. Annual Report of the Office of the Auditor General of Ontario, Hospital Emergency Departments. Ottawa: Canadian Ministry of Health and Long Term Care; 2010. Available at: http://www.auditor.on. ca/en/content/annualreports/arreports/en12/405en12.pdf (accessed June 4, 2017).

5. Canadian Institute for Health Information. Sources of Potentially Avoidable Emergency Department Visits. Ottawa: Canadian Institute for Health Information; 2014. Available at: https://secure.cihi.ca/free_products/ED_ Report_ForWeb_EN_Final.pdf (accessed June 4, 2017).

6. Taheri PA, Butz D, Griffes LC, Morlock DR, Greenfield LJ. Physician impact on the total cost of care. Ann Surg 2000;231(3):432-5.

7. Nougon G, Muschart X, Gerard V, et al. Does offering pricing information to resident physicians in the emergency department potentially reduce laboratory and radiology costs. Eur 7 Emerg Med 2015;22(4):247-52.

8. Covington MF, Agan DL, Liu Y, Johnson JO, Shaw DJ. Teaching cost-conscious medicine: Impact of a simple educational intervention on appropriate abdominal imaging at a community-based teaching hospital. $7 \mathrm{Grad}$ Med Educ 2013;5(2):284-8.

9. Calderon-Margalit R, Mor-Yosef S, Mayer M, Adler B, Shapira SC. An administrative intervention to improve the utilization of laboratory tests within a university hospital. Int 7 Qual Health Care 2005;17(3):243-8.

10. Attali M, Barel Y, Somin M, et al. A cost-effective method for reducing the volume of laboratory tests in a university-associated teaching hospital. Mt Sinai $7 \mathrm{Med}$ 2006;73(5):787-94.

11. Larsson A, Blom S, Wernroth ML, Hultén G, Tryding N. Effects of an education programme to change clinical laboratory testing habits in primary care. Scand 7 Prim Health Care 1999;17(4):238-43.

12. Agarwal N, Agarwal P, Mazurkiewicz A, Wecht DA, Friedlander RM. Neurosurgical cost containment via improved physician awareness. Neurosurgery 2016;63 (Suppl 1):194 doi:10.1227/01.neu.0000489813.98347.20.

13. Allan GM, Lexchin J, Wiebe N. Physician awareness of drug cost: a systematic review. PLoS Med 2007;4(9):e283.

14. Allan GM, Innes G. Family practice residents' awareness of medical care costs in British Columbia. Fam Med 2002; 34(2):104-9.

15. Allan GM, Lexchin J. Physician awareness of diagnostic and nondrug therapeutic costs: a systematic review. Int 7 Technol Assess Health Care 2008;24(2):158-65.

16. Vijayasarathi A, Duszak R, Gelbard RB, Mullins ME. Knowledge of the costs of diagnostic imaging: a survey of physician trainees at a large academic medical center. $7 \mathrm{Am}$ Coll Radiol 2016;13(11):1304-10.

17. DeMarco SS, Paul R, Kilpatrick RJ. Information system technologies' role in augmenting dermatologists' knowledge of prescription medication costs. Int 7 Med Inform 2015; 84(12):1076-84.

18. Schilling UM. The acceptance of price lists at the emergency department: how do doctors think about it. Scand $\mathcal{f}$ Trauma Resusc Emerg Med 2010;18(Suppl 1):32.

19. Long T, Bongiovanni T, Dashevsky M, et al. Impact of laboratory cost display on resident attitudes and knowledge about costs. Postgrad Med 7 2016;92(1092):592-6.

20. Lysdahl KB, Hofmann BM. What causes increasing and unnecessary use of radiological investigations? A survey of radiologists' perceptions. BMC Health Serv Res 2009;9:155.

21. Kinch MS, Haynesworth A, Kinch SL, Kinch SL, Hoyer D. An overview of FDA-approved new molecular entities: 1827-2013. Drug Discov Today 2014;19(8):1033-9.

22. Long MJ, Cummings KM, Frisof KB. The role of perceived price in physicians' demand for diagnostic tests. Med Care 1983;21(2):243-50.

23. Innes G, Grafstein E, McGrogan J. Do emergency physicians know the costs of medical care. CFEM 2000;2(2): 95-102.

24. Cyriac JM, James E. Switch over from intravenous to oral therapy: a concise overview. I Pharmacol Pharmacother 2014;5(2):83-7.

25. Wright JM, Price SD, Watson WA. NSAID use and efficacy in the emergency department: single doses of oral ibuprofen versus intramuscular ketorolac. Ann Pharmacother 1994; 28(3):309-12.

26. Chou R, Qaseem A, Snow V, et al. Diagnosis and treatment of low back pain: a joint clinical practice guideline from the American College of Physicians and the American Pain Society. Ann Intern Med 2007;147(7):478-91. 


\section{APPENDIX}

Sample Survey Question

\section{Cost Survey}

2. Costs of Imaging (1/4)

$2 / 5$

$40 \%$

1. Please estimate the total direct cost to $\mathrm{TOH}$ (in 2014), for performing one of these imaging tests.

('Total direct cost' includes: direct fixed costs (overhead, technician salary, etc.) and any variable costs (materials, imaging films, etc.).

2. Do not include radiologist interpretation costs.

You can round to the nearest dollar or include decimals for each question

If you are unsure, please provide your best estimate

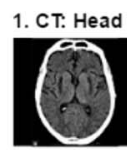

$\$$

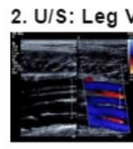

$\$$
3. X-Ray: Abdomen/KUB (2 View)

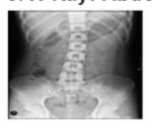

